\title{
Experimental Violation of Local Realism by Four-photon Greenberger-Horne-Zeilinger Entanglement
}

\author{
Zhi Zhao, ${ }^{1}$ Tao Yang, ${ }^{1}$ Yu-Ao Chen, ${ }^{1}$ An-Ning Zhang, ${ }^{1}$ Marek Żukowski, ${ }^{2}$ and Jian-Wei Pan ${ }^{1,3}$ \\ ${ }^{1}$ Department of Modern Physics, University of Science and Technology of China, \\ Hefei, Anhui 230027, People's Republic of China \\ ${ }^{2}$ Instytut Fizyki Teoretycznej $i$ Astrofizyki Uniwersytet Gdanski, PL-80-952 Gdansk, Poland \\ ${ }^{3}$ Institut für Experimentalphysik, Universitat Wien, Boltzmanngasse 5, 1090 Wien, Austria
}

\begin{abstract}
We report the first experimental violation of local realism in four-photon Greenberger-HorneZeilinger (GHZ) entanglement. In the experiment, the non-statistical GHZ conflicts between quantum mechanics and local realism are confirmed, within the experimental accuracy, by four specific measurements of polarization correlations between four photons. In addition, our experimental results not only demonstrate a violation of Mermin-Ardehali-Belinskii-Klyshko inequality by 76 standard deviations, but also for the first time provide sufficient evidence to confirm the existence of genuine four-particle entanglement.
\end{abstract}

PACS numbers: 03.65.Ud, 03.67.Mn,42.50.Dv

Multi-particle entanglement not only plays a crucial role in fundamental tests of quantum mechanics (QM) versus local realism (LR), but is also at the basis of nearly all quantum information protocols such as quantum communication and quantum computation [1]. Since the seminal work of Greenberger, Horne, Zeilinger (GHZ) [2], the research on multi-particle entanglement has received much attention. In contrast to the case of twoparticle entanglement where only statistical correlation predicted by QM is inconsistent with LR, in the case of maximally entangled states of more than two particles (i.e. the so-called GHZ states) a conflict with LR arises even for nonstatistical predictions of QM 2]. Further, QM can violate the multi-particle Bell-type inequalities imposed by LR by an amount that grows exponentially with the number of entangled particles $3,[4,5,[6]$, that is, going to higher entangled systems the conflict between $\mathrm{QM}$ and LR becomes ever stronger.

In recent years, entanglement of three photons has been realized experimentally [7] and used to demonstrate the extreme GHZ contradiction between QM and LR 8]. Meanwhile, entanglement of three atoms $[9]$ or four ions 10 has also been demonstrated, yet in these two cases the quality of the entangled states still needs to be improved significantly in order to be useful for tests of LR and for quantum information processing. Though significant experimental progress has been achieved, all the above experiments suffer from a loophole in confirming genuine multi-particle entanglement 11. This is due to the fact that the data measured in any of the above $\mathrm{N}$ particle entanglement experiments can be explained by a hybrid model in which only less than $N$ particles are entangled 11]. Using the highly pure four-photon entanglement achieved in a recent experiment [12], it is, in principle, possible to exclude such a hybrid model by showing a sufficient violation of Bell-type inequalities. However, due to the very low coincidence rate in the experiment it was not possible to show such a violation. Therefore, the loophole problem still remains unsolved.

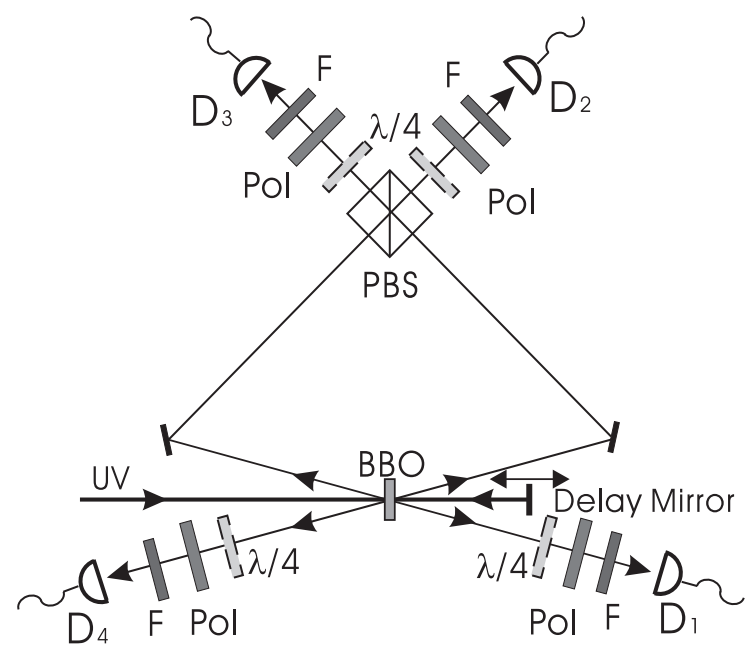

FIG. 1: Experimental setup for observing high intensity fourphoton GHZ entanglement. Two pairs of entangled photons are produced by passing a UV laser pulse through a BBO crystal twice. The UV laser with a central wavelength of $394 \mathrm{~nm}$ has a pulse duration of $200 \mathrm{fs}$, a repetition rate of $76 \mathrm{MHz}$ and an average pump power of $450 \mathrm{~mW}$. By optimizing the collection efficiency [14], we are able to observe about $2 \times 10^{4}$ entangled pairs per second for each pair behind 3.6nm filters $(\mathrm{F})$ of central wavelength $788 \mathrm{~nm}$. Coincidences between detectors $D_{1}, D_{2}, D_{3}$ and $D_{4}$ exhibit four-photon GHZ entanglement. Polarizers (POL) and quarter wave-plates $(\lambda / 4)$ are used to perform measurement of linear $H^{\prime} / V^{\prime}$ or $R / L$ polarization.

In this Letter, we develop a high intensity source of four-photon GHZ entanglement 13], by which we report the first four-observer test of GHZ contradiction, and for the first time provide sufficient experimental evidence to confirm the existence of genuine four-particle entanglement, hence closing the possible loophole of a hybrid model.

To demonstrate the four-photon GHZ contradiction, we first generate four-photon entanglement using the 


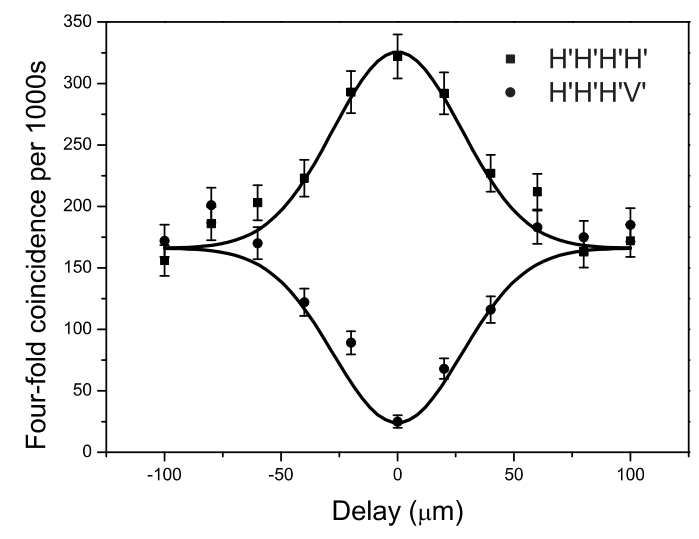

FIG. 2: Typical experimental results for polarization measurements on all four photons in the $H^{\prime} / V^{\prime}$ basis. The coincidence rates of $H^{\prime} H^{\prime} H^{\prime} H^{\prime}$ and $H^{\prime} H^{\prime} H^{\prime} V^{\prime}$ components are shown as a function of the pump delay mirror position. The high visibility obtained at zero delay implies that four photons are indeed in a coherent superposition.

technique developed in a previous experiment 12]. As shown in Fig.1, a pulse of ultraviolet (UV) light passes through a BBO crystal twice to produce two polarizationentangled photon pairs. One photon out of each pair is then steered to a polarization beam splitter (PBS) where the path lengths of each photon have been adjusted such that they arrive simultaneously. After the two photons pass through the PBS, correlations due to four-photon GHZ entanglement,

$$
|\Psi\rangle=\frac{1}{\sqrt{2}}\left(|H\rangle_{1}|V\rangle_{2}|V\rangle_{3}|H\rangle_{4}+|V\rangle_{1}|H\rangle_{2}|H\rangle_{3}|V\rangle_{4}\right),
$$

can thus be observed, if all four detectors click (see [15]). Here $H(V)$ denotes horizontal (vertical) linear polarization.

The observed four-fold coincident rate of the desired component $H V V H$ or $V H H V$ was about 1.3 per second, which is almost two orders of magnitude brighter than the previous experiment 12]. The ratio between any of the desired four-fold events $H V V H$ and $V H H V$ to any of the 14 other nondesired ones is better than $60: 1$. To confirm the state (11) is indeed in a coherent superposition, we have performed polarization measurements on the four photons in the $H^{\prime} / V^{\prime}$ basis, where $\left|H^{\prime}\right\rangle=1 / \sqrt{2}(|H\rangle+|V\rangle)$ and $\left|V^{\prime}\right\rangle=1 / \sqrt{2}(|H\rangle-|V\rangle)$. In Fig. 2, we compare the count rates of $H^{\prime} H^{\prime} H^{\prime} H^{\prime}$ and $H^{\prime} H^{\prime} H^{\prime} V^{\prime}$ components as we move the delay mirror. At zero delay, the latter component is suppressed with a visibility of $0.84 \pm 0.03$, hence confirming the coherent superposition of $H V V H$ and $V H H V$. The high visibility and high intensity achieved indicates that the source is good enough to demonstrate the GHZ contradiction between QM and LR.
Let us analyze the QM predictions for the four-photon state (1). Since the polarization states of a photon are a realization of a qubit, one can represent $|H\rangle$ by column vector $\left(\begin{array}{l}0 \\ 1\end{array}\right)$ and $|V\rangle$ by column vetcor $\left(\begin{array}{l}1 \\ 0\end{array}\right)$. That is, they can be treated as the two eigenvectors of Pauli operator $\sigma_{x}$ of eigenvalues +1 and -1 respectively. Adopting the methods of Refs. 8, 16], we consider measurements of linear polarization $H^{\prime} / V^{\prime}$, or of circular polarization $R / L$, where $|R\rangle=\frac{1}{\sqrt{2}}(|H\rangle+i|V\rangle)$ and $|L\rangle=\frac{1}{\sqrt{2}}(|H\rangle-i|V\rangle)$ can be represented as the two eigenstates of Pauli operator $\sigma_{y}$ with eigenvalues \pm 1 . We shall call a measurement of $H^{\prime} / V^{\prime}$ linear polarization as a $\sigma_{x}$ measurement and one of $R / L$ circular polarization as a $\sigma_{y}$ measurement.

To illustrate the GHZ conflict between QM and LR, we first consider three specific measurements of polarization correlations between four photons:

$$
\sigma_{x} \sigma_{x} \sigma_{x} \sigma_{x}, \sigma_{x} \sigma_{y} \sigma_{x} \sigma_{y}, \sigma_{x} \sigma_{x} \sigma_{y} \sigma_{y}
$$

where, for example, $\sigma_{x} \sigma_{x} \sigma_{y} \sigma_{y}$ denotes a joint measurement of linear polarization $H^{\prime} / V^{\prime}$ on photons 1 and 2, and circular polarization $R / L$ on photons 3 and 4 . The three operators in Eq. (2) commute with each other and the state (11) is their common eigenstate with the eigenvalue +1 . Thus, in any of the three measurements, the total number of photons that carry either $V^{\prime}$ or $L$ polarization (i.e. with eigenvalue -1) must be even. For example, in a $\sigma_{x} \sigma_{x} \sigma_{y} \sigma_{y}$ measurement, only polarization combinations $H^{\prime} H^{\prime} R R, H^{\prime} H^{\prime} L L, H^{\prime} V^{\prime} R L, H^{\prime} V^{\prime} L R, V^{\prime} H^{\prime} R L$, $V^{\prime} H^{\prime} L R, V^{\prime} V^{\prime} R R$, and $V^{\prime} V^{\prime} L L$ arise. Similar constraints can also be obtained for the other two measurements of (2).

We now analyze what are the implications for LR. Although any specific result obtained in any joint measurement on less than four photons is maximally random, one can still presume that, each photon carries EinsteinPodolsky-Rosen (EPR) elements of reality 17] for both $\sigma_{x}$ and $\sigma_{y}$ measurements that determine the specific individual measurement result [18]. This is because in every one of the three measurements, any individual measurement result - both for circular polarization and for linear polarization $H^{\prime} / V^{\prime}$ - can be predicted with certainty for every photon given the corresponding measurement results of the other three [8, 16].

For any photon $i$ we call these elements of reality $X_{i}$ with values $+1(-1)$ for $H^{\prime}\left(V^{\prime}\right)$ polarizations and $Y_{i}$ with values $+1(-1)$ for $R(L)$; we thus obtain the relations $X_{1} X_{2} X_{3} X_{4}=X_{1} Y_{2} X_{3} Y_{4}=X_{1} X_{2} Y_{3} Y_{4}=+1$, in order to be able to reproduce the quantum predictions on all three measurements in Eq. (2). Furthermore, according to LR, any specific measurement for $\sigma_{x}$ or $\sigma_{y}$ must be independent of whether a $\sigma_{x}$ or $\sigma_{y}$ measurement is performed on the other photons. As $X_{i} X_{i}=+1$ and $Y_{i} Y_{i}=+1$, we can write $X_{1} Y_{2} Y_{3} X_{4}=\left(X_{1} X_{2} X_{3} X_{4}\right)\left(X_{1} Y_{2} X_{3} Y_{4}\right)\left(X_{1} X_{2} Y_{3} Y_{4}\right)$ and obtain $X_{1} Y_{2} Y_{3} X_{4}=+1$. 
(a)

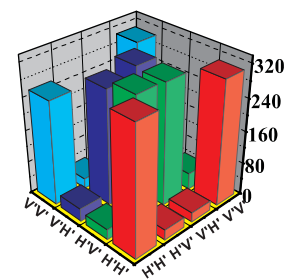

(b)

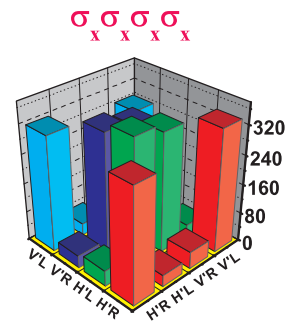

(c)

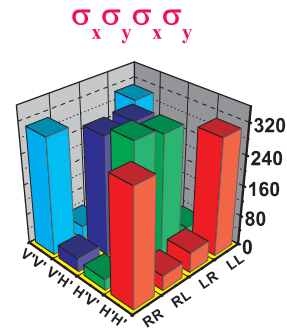

$\sigma_{x} \sigma_{x} \sigma_{y} \sigma_{y}$ (d)

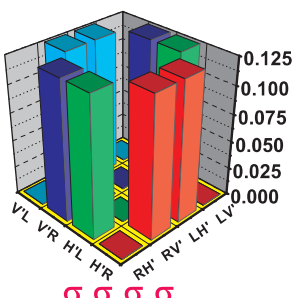

(e)

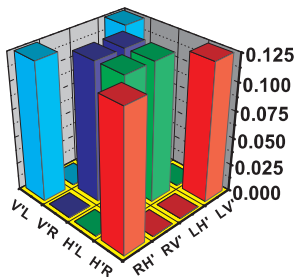

(f)

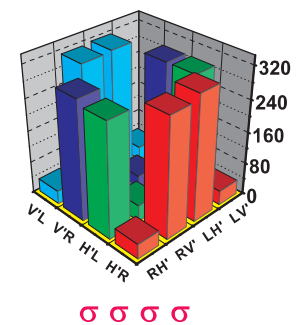

FIG. 3: Experimental results observed in the first three experiments (a)-(c), and predictions of QM and of LR (normalized), and observed results for the $\sigma_{x} \sigma_{y} \sigma_{y} \sigma_{x}$ measurement (d)-(f). The visibilities in (a)-(c) are $0.820 \pm 0.011,0.807 \pm 0.011$ and $0.781 \pm 0.012$, respectively. The experimental results in (f) are in agreement with the QM predictions (d) while in conflict with LR (e), with a visibility of $0.789 \pm 0.012$. The integration time of each four-fold coincidence is 1000s.

Therefore, the existence of the elements of reality implies that, performing a $\sigma_{x} \sigma_{y} \sigma_{y} \sigma_{x}$ measurement on the state (11), one should obtain the product of the eigenvalues with +1 . Thus, from a local realistic point of view the only possible results for a $\sigma_{x} \sigma_{y} \sigma_{y} \sigma_{x}$ measurement are $H^{\prime} R R H^{\prime}, H^{\prime} R L V^{\prime}, H^{\prime} L R V^{\prime}, H^{\prime} L L H^{\prime}, V^{\prime} R R V^{\prime}$, $V^{\prime} R L H^{\prime}, V^{\prime} L R H^{\prime}$, and $V^{\prime} L L V^{\prime}$ (as shown in Fig. 3e).

However, according to QM, the state (11) is an eigenstate with eigenvalue -1 for operator $\sigma_{x} \sigma_{y} \sigma_{y} \sigma_{x}$. Thus, QM predicts that the only possible results for a $\sigma_{x} \sigma_{y} \sigma_{y} \sigma_{x}$ measurement are $H^{\prime} R R V^{\prime}, H^{\prime} R L H^{\prime}, H^{\prime} L R H^{\prime}, H^{\prime} L L V^{\prime}$, $V^{\prime} R R H^{\prime}, V^{\prime} R L V^{\prime}, V^{\prime} L R V^{\prime}$, and $V^{\prime} L L H^{\prime}$ (as shown in Fig. 3d). Thus we conclude that the predictions by LR is completely opposite to the predictions by QM and vice versa. It is the GHZ contradiction between LR and QM that exhibits a more powerful refutation of the existence of elements of reality than the one provided by Bell's theorem for two-particle entanglement.

To demonstrate experimentally this conflict between LR and QM, we perform four polarization correlation measurements on the four spatially separated photons.

The observed results for the first three measurements are shown in Figs. 3a, 3b, 3c. Each measurement consists of 16 possible outcomes and ideally only eight of them should occur. However, since in reality no experiment can ever be perfect, even the outcomes which should not occur will occur with some small probabilities. Thus, if we are allowed to assume that the spurious events are attributable to the unavoidable experimental errors, then within the experimental accuracy we can conclude that the desired correlations in the three measurements confirm the quantum predictions for our GHZ entanglement.

In Figs. 3d, 3e, 3f , we compare the predictions of QM and LR with the results of the fourth $\sigma_{x} \sigma_{y} \sigma_{y} \sigma_{x}$ measurement. The results show that, within experimental error, the four-fold coincidences predicted by QM occur, and not those predicted by LR. In this sense, we claim that we have experimentally realized the first four-particle test of local realism following the GHZ argument. For the purists, we may note that there is a derivation of the GHZ paradox for situations involving up to $25 \%$ (data flipping) error rate [19], that is for rates much higher than observed in the experiment $(\sim 11 \%)$.

The conflict between the quantum predictions for the GHZ states and local realism can also be shown via violation of a suitable Bell inequality. In this case taking account of the errors is straightforward. A number of inequalities for $N$-particle GHZ states have been derived $[\underline{3}, 4,[5,[6]$. According to the optimal MABK inequality for four-particle GHZ state [4], LR imposes a constraint on statistical correlations of polarization measurements on the four-photon system as the following:

$$
|\langle A\rangle| \leq 2
$$

where

$$
\begin{aligned}
A & =\frac{1}{2}\left(\sigma_{x} \sigma_{x} \sigma_{x}-\sigma_{x} \sigma_{y} \sigma_{y}+\sigma_{y} \sigma_{x} \sigma_{y}+\sigma_{y} \sigma_{y} \sigma_{x}\right)\left(\sigma_{a}+\sigma_{b}\right) \\
& +\frac{1}{2}\left(\sigma_{y} \sigma_{y} \sigma_{y}-\sigma_{x} \sigma_{y} \sigma_{x}+\sigma_{x} \sigma_{x} \sigma_{y}+\sigma_{y} \sigma_{x} \sigma_{x}\right)\left(\sigma_{a}-\sigma_{b}\right)
\end{aligned}
$$

and $\sigma_{a}=\frac{1}{\sqrt{2}}\left(\sigma_{x}+\sigma_{y}\right), \sigma_{b}=\frac{1}{\sqrt{2}}\left(\sigma_{x}-\sigma_{y}\right)$, and they correspond to measurements of two (othogonal) pairs of elliptic polarizations. In Eq. (3), for example, $\left\langle\sigma_{x} \sigma_{x} \sigma_{x} \sigma_{a}\right\rangle$ denotes the expectation value of a $\sigma_{x} \sigma_{x} \sigma_{x} \sigma_{a}$ measurement on the four photons. QM predicts a maximal violation of the constraint by a factor of $2 \sqrt{2}$. For the prefect quantum prediction the visibility of the correlations can be reduced to as little as $35.4 \%$. Interestingly, a different set of measurements, than those for the GHZ contradiction, are optimal in the case of this inequality. Further, one could note that the inequalities derived in [ $[\underline{]}$ ] require only a visibility of $32.9 \%$. All this should be contrasted with the visibility consistent with the result of ref. [19], concerning the GHZ contradiction, which is $50 \%$. Therefore in order to get maximal possible disagreement with LR, we performed another set of measurements.

To measure the expectation value of $A$, we need to perform sixteen specific measurements such as 
$\sigma_{x} \sigma_{x} \sigma_{x} \sigma_{a}, \ldots, \sigma_{y} \sigma_{x} \sigma_{x} \sigma_{b}$. A $\sigma_{a}$ measurement on photon 4 is obtained if we insert in its path a quarter wave plate (QWP), whose optical axis is set at $45^{\circ}$ with respect to the horizontal direction. Then, the two eigenstates of operator $\sigma_{a}$ are converted into linear polarizations which are polarized along the directions of $-22.5^{\circ}$ and $67.5^{\circ}$. In the same way, the two eigenstates of operator $\sigma_{b}$ can be converted into $-67.5^{\circ}$ and $22.5^{\circ}$ linear polarizations. The average visibility observed in the experiment for the state (1) is $78.4 \%$ and thus greatly exceed the minimum of $35.4 \%$. Substituting the experimental results into the left-hand side of inequality (3) gives

$$
|\langle A\rangle|=4.433 \pm 0.032
$$

which violate the inequality (3) by over 76 standard deviations, hence demonstrating the conflict between QM and LR in four-photon GHZ entanglement.

Furthermore, the high visibilities also confirm the existence of genuine four-photon entanglement in our experiment. To demonstrate a full four-photon entanglement, two sufficient conditions, i.e. the inequality $|\langle A\rangle|>4$ and the so-called state preparation fidelity $F(\rho)>1 / 2$, must be satisfied [11, 20]. Here the state preparation fidelity is defined as

$$
\begin{aligned}
F(\rho) & =\langle\Psi|\rho| \Psi\rangle \\
& =\frac{1}{2}(\langle H V V H|\rho| H V V H\rangle+\langle V H H V|\rho| V H H V\rangle) \\
& +\operatorname{Re}\langle H V V H|\rho| V H H V\rangle
\end{aligned}
$$

and for any state $\rho$, there is a simple identity:

$$
|\langle A\rangle|=8 \sqrt{2} \operatorname{Re}\langle H V V H|\rho| V H H V\rangle .
$$

Not only does the experimental result in Eq. (5) significantly violate the inequality $|\langle A\rangle|>4$, together with the observed fractions of the desired components and the nondesired ones in the $H / V$ basis it also gives $F(\rho)=0.840 \pm 0.007$, which is well above the threshold of $1 / 2$. Thus, our experiment for the first time provides unambiguous evidence for a full test of four-particle entanglement, which excludes any hybrid hidden-variable model to explain our experimental data.

In conclusion, we have demonstrated the statistical and nonstatistical conflicts between QM and LR in fourphoton GHZ entanglement. However, it is worth noting that, as for all existing photonic tests of LR, we also had to invoke the fair sampling hypothesis due to the very low detection efficiency in our experiment. Possible future experiments could include further study of GHZ correlations over large distances with space-like separated randomly switched measurements [21]. Our work, besides its significance in quantum foundations, could also be applied to investigate the basic elements of quantum computation with linear optics [22] and implement multiphoton quantum secrete sharing [23].

This work was supported by the National Natural Science Foundation of China, Chinese Academy of Sciences and the National Fundamental Research Program (under Grant No. 2001CB309303). MZ is supported by the UG grant BW-5400-5-0256-3.
[1] D. Bouwmeester, A. Ekert, and A. Zeilinger, The Physics of Quantum Information, (Spinger, Berlin 2000).

[2] D. M. Greenberger, M. Horne, and A. Zeilinger, in Bell's Theorem, Quantum Theory, and Conceptions of the universe, edited by M. Kafatos (Kluwer Academic, Dordrecht, 1989), pp. 69-72.

[3] N. D. Mermin, Phys. Rev. Lett. 65, 1838 (1990).

[4] M. Ardehali, Phys. Rev. A 46, 5375 (1992).

[5] A. V. Belinskii and D. N. Klyshko, Phys. Usp. 36, 653 (1993).

[6] M. Żukowski, Phys. Lett. A 177, 290 (1993).

[7] D. Bouwmeester et al., Phys. Rev. Lett. 82, 1345 (1999).

[8] J.-W. Pan et al., Nature (London) 403, 515 (2000).

[9] A. Rauschenbeutel et al., Science 288, 2024 (2000).

[10] C. A. Sackett, et al., Nature (London) 404, 256 (2000).

[11] M. Seevinck and J. Uffink, Phys. Rev. A 65, 012107 (2001); J. Uffink, Phys. Rev. Lett. 88, 230406 (2002); M. Seevinck and G. Svetlichny, ibid 89, 060401 (2002).

[12] J.-W. Pan et al., Phys. Rev. Lett. 86, 4435 (2001).

[13] In a recent experiment, four-photon correlations of a different type than the GHZ ones were observed by M. Eibl et al., quant-ph/0302042

[14] C. Kurtsiefer, M. Oberparleiter, and H. Weinfurter,
Phys. Rev. A 64, 023802 (2001).

[15] A. Zeilinger, M. A. Horne, H. Weinfurter, M. Zukowski, Phys. Rev. Lett. 78, 3031 (1997).

[16] N. D. Mermin, Phys. Today 43, 9 (1990).

[17] A. Einstein, B. Podolsky, N. Rosen, Phys. Rev. 47, 777 (1935).

[18] Note that, to argue, that photon 1 also carries element of reality for a $\sigma_{y}$ measurement, we could consider a $\sigma_{y} \sigma_{y} \sigma_{y} \sigma_{y}$ measurement for which the state (1) is again an eigenstate with eigenvalue +1 .

[19] L. C. Ryff., Am.J. Phys. 65(12), 1197-1199 (1997)

[20] N. Gisin, H. Bechmann-Pasquinucci, Phys. Lett. A 246, 1 (1998).

[21] G. Weihs et al., Phys. Rev. Lett. 81, 5039 (1998).

[22] E. Knill, R, Laflamme, and G. Milburn, Nature (London) 409, 46 (2001).

[23] M. Żukowski, A. Zeilinger, M.A. Horne and H. Weinfurter, Acta Phys. Pol. 93, 187 (1998). M. Hillery, V. Buzek, and A. Berthiaume, Phys. Rev. A 59, 1829 (1999); V. Scarani and N. Gisin, Phys. Rev. Lett. 87, 117901 (2001). 